\title{
Descripción de los estatolitos y relaciones morfométricas y gravimétricas en el calamar patagónico (Loligo gahi ) (Cephalopoda: Loliginidae).
}

\author{
Statoliths descriptions and morphometrics and gravimetrics relationship in \\ patagonian squid (Loligo gahi) (Cephalopoda: Loliginidae). \\ Lorenzo A. Flores y David E. Garland. \\ Universidad de Valparaíso, Facultad de Ciencias del Mar. Av Borgoño s/n, Reñaca, casilla 13-D Viña del Mar, Chile. \\ lorenzo.flores@uvalparaiso.cl
}

\begin{abstract}
Resumen.- Se realizó la descripción de los estatolitos y la determinación de relaciones morfométricas y gravimétricas en el calamar patagónico Loligo gahi a partir de una muestra provienente de la captura desarrollada entre Enero y Febrero de 1997 en la pesquería comercial del sur de las íslas Falkland. Para realizar el estudio la muestra total de 305 individuos fue submuestrada aleatoriamente a través de estratos de pesos. Morfológicamente los estatolitos, presentan un domo dorsal y lateral bien desarrollado y un rostro pequeño con su punta curvada. La relación entre la longitud dorsal del manto (LM) y la longitud del estatolito (LE) está representada por un modelo geométrico que mostró un crecimiento alométrico ( $\mathrm{b}=0.208)$. La relación longitud del manto (LM) y peso (P) correspondió también a un modelo geométrico de tipo alométrico ( $\mathrm{b}=2.388$ ). Palabras Claves: Calamar, Loligo gahi, estatolitos, relaciones morfométricas, relaciones gravimetricas, islas Falkland.
\end{abstract}

\begin{abstract}
The statolith of the patagonian squid (Loligo gahi) was described and the morphometrics and gravimetrics relationship were determined. The squid was caught at the south of Falkland island during the summer of 1997. The 305 squid were subsampled using a stratified random sampled of weight of squid. The statolith of patagonian squid presents a well developed dorsal and lateral dome and the rostrum is small and curved. The morphometrics relationship between dorsal length of mantle and statolith length was described by a geometric mathematical model and shows an allometric growth $(b=0.208)$. The gravimetrics relationship between dorsal length of mantle and weigth of patagonian squid was also described by a geometric mathemetical model showing an allometric growth $(\mathrm{b}=2.388)$.

Key words: Squid, Loligo gahi, statoliths, morphometrics relationship, gravimetrics relationship, Falkland islands.
\end{abstract}

\section{Introducción}

Loligo gahi (d' Orbigny, 1835) es un calamar miópsido que se encuentra en aguas templadas de la plataforma y talud de las costas Atlántica y Pacífica de Sudamérica desde el sur del Perú hasta Argentina y sustenta una de las mayores pesquerías internacionales sobre la plataforma Patagónica siendo capturado comercialmente por la flota internacional dentro de la Zona de Conservación y manejo Interino de las Islas Falklands (Roper et al.1984, Csirke 1987). La pesquería en la zona de Islas Falklands captura anualmente cerca de 700.000 toneladas de cefalópodos, de las cuales 50.000 a 70.000 toneladas corresponden a las capturas de Loligo gahi (FAO 1998).

Debido a que los métodos utilizados más frecuentemente en estimación de índices de crecimiento y edad a través del análisis de datos de frecuencia de longitud no son aplicables a cefalópodos (Natsukari et al. 1988, Arkhipkin 1991, Caddy 1991, Arkhipkin \&
Mikheev 1992, Jackson 1994) es que en años recientes los estatolitos han llegado a ser la principal herramienta en estudios de determinación de edad en calamares. Los estatolitos de los cefalópodos son estructuras calcáreas pares compuestos por carbonato de calcio en su forma de aragonita de poca transparencia y consistencia dura que se alojan en los estatocistos y su posición es ventroposterior al cerebro. Su función es el mantenimiento del equilibrio, control de la aceleración lineal, rotación y balanceo del cuerpo (Clarke 1978, Leta 1982, Rodhouse \& Hatfield 1990, Hatfield 1991, Jackson 1994). Los estatolitos tienen valor taxonómico y son útiles en establecer relaciones entre familias y géneros (Clarke 1978, Leta 1982, Brunetti \& Ivanovic 1990). En ecología trófica el estudio de la morfología de los estatolitos de cefalópodos presentes en el contenido estomacal de especies predatorias de dichos recursos, resulta especialmente útil para fines de identificación, (Clarke 1975, Clarke 1978, Leta 1982; Collins \& Pierce 1996, Pineda et al. 1998, Vega et al. 2001). Sin embargo hasta el presente no son numerosos los estudios de la morfología en los estatolitos de 
loligínidos. Clarke (1978) entrega definiciones y dimensiones en un estatolito tipo y describe los estatolitos de Loligo forbesi; Leta (1982) describe los estatolitos de Loligo bransiliensis; Pineda et al. (1998) comparan los estatolitos de Loligo sanpaulensis y Loligo gahi del Atlántico suroccidental.

Cuando un animal crece su forma en cualquier momento es el resultado del aumento en talla y volumen que es medido como variables de longitud y peso respectivamente, siendo la estimación de esta relación norma en estudios pesqueros, pudiendo variar en una misma especie según el sexo, estación del año y ubicación geográfica (Ricker 1979, Forsythe \& Van Heukelem 1987). Esta relación se ha estimado en calamares neríticos como Loligo forbesi (Holme 1974), Loligo gahi (Arancibia \& Robotham 1984), Loligo forbesi (Collins et al. 1995), Loligo vulgaris (Raya et al. 1999). Otra relación importante es la de proporcionalidad entre la longitud del manto (LM) y la longitud del estatolito (LE). Cuando el valor de la pendiente en la relación LM/LE es diferente a 1 , se tiene un crecimento alométrico, lo que significa que la tasa de crecimiento del calamar es diferente a la tasa de crecimiento del estatolito (Ricker 1979). En loligínidos esta relación de crecimiento tiene pocos antecedentes en la literatura, sin embargo ha sido estimada para Loligo gahi (Hatfield 1991), Loligo vulgaris (Arkhipkin 1995), Loligo forbesi (Collins et al. 1995), Alloteuthis subulata, A. africana (Arkhipkin \& Nekludova 1993).

Este artículo tiene por objeto describir la morfología de los estatolitos, determinar la relación morfométrica entre la longitud del manto versus la longitud del estatolito, y la gravimétrica entre la longitud del manto versus el peso en Loligo gahi.

\section{Materiales y Métodos}

Se utilizó una muestra del calamar patagónico Loligo gahi, capturado 50 millas al sur de las Islas Falkland, con una red de arrastre de media agua en la última marea de 1997 del B/F “Betanzo” de Pesca Chile S.A. Los calamares se midieron considerando la longitud total y la del manto con una regla milimetrada y se pesaron en una balanza Sartorius - Werke tipo 2254 con una precisión de 0,01g. Para describir los estatolitos y determinar las relaciones morfométricas y gravimétricas se realizó un submuestreo aleatorio estratificado con asignación óptima (Cochran 1971) a partir de la distribución de frecuencia de pesos de la muestra total $(\mathrm{N}=305)$ sin diferenciar entre machos y hembras con un rango entre 9,67g y 63,69 g.
La descripción de los estatolitos de Loligo gahi se realizó con el par derecho de las submuestras $(n=47)$ provenientes de todos los estratos de pesos. En La descripción morfológica se empleó las terminologías propuestas por Clarke (1978), Leta (1982) y Lipinski et al. (1991). Los estatolitos fueron fotografiados en diferentes planos con una película pancromática de 100 asas, a través de una cámara Leica Wild MPS12 montada en una lupa binocular Leica Wild M8.

A partir de los datos de longitud del manto y peso de los individuos pertenecientes a la submuestra se ajustó una relación matemática asignando como variable independiente la longitud del manto y como variable dependiente el peso total. La relación matemática entre la longitud del manto y la longitud del estatolito, se determinó asignando como variable independiente la longitud del manto y como variable dependiente la longitud del estatolito, que fue medida desde el rostro hasta el domo dorsal. Para ambas relaciones el análisis estadístico de las variables fue realizado a través de la rutina de regresión simple del programa Statistica versión 6.0 determinando el modelo más adecuado en función del coeficiente de determinación, error estándar y análisis de los residuos.

\section{Resultados}

\section{Descripción de los estatolitos en Loligo gahi}

Los estatolitos en los calamares están formados por cuatro zonas principales que son el domo dorsal, el domo lateral, el rostro y el ala. Las tres primeras son de consistencia dura (aragonita), a diferencia del ala, que es muy frágil (calcita), especialmente por el lado anterior que puede destruirse cuando los estatolitos son extraidos (Clarke 1978). En las figuras 1A y 1B se observa un estatolito con sus partes según la clasificación empleada por Clarke (1978) y Leta (1982). Los distintos planos de orientación de un estatolito se observan en la figura 1C según Lipinski et al. (1991).

Los estatolitos observados en la submuestra $(n=47)$ incluyeron individuos entre los 9,67 g y 63,64 g de peso con longitudes de los estatolitos entre 1,36 mm y 1,57 $\mathrm{mm}$, no mostrando diferencias morfológicas importantes a través de los pesos, observándose una forma característica. Así los estatolitos de Loligo gahi fueron descritos de la siguiente manera:

El domo dorsal es cóncavo por su lado anterior y convexo en su parte posterior y pequeño respecto al resto del estatolito. Es redondeado anteroposteriormente y de textura lisa. La separación entre el domo dorsal y el domo lateral esta dada por una 

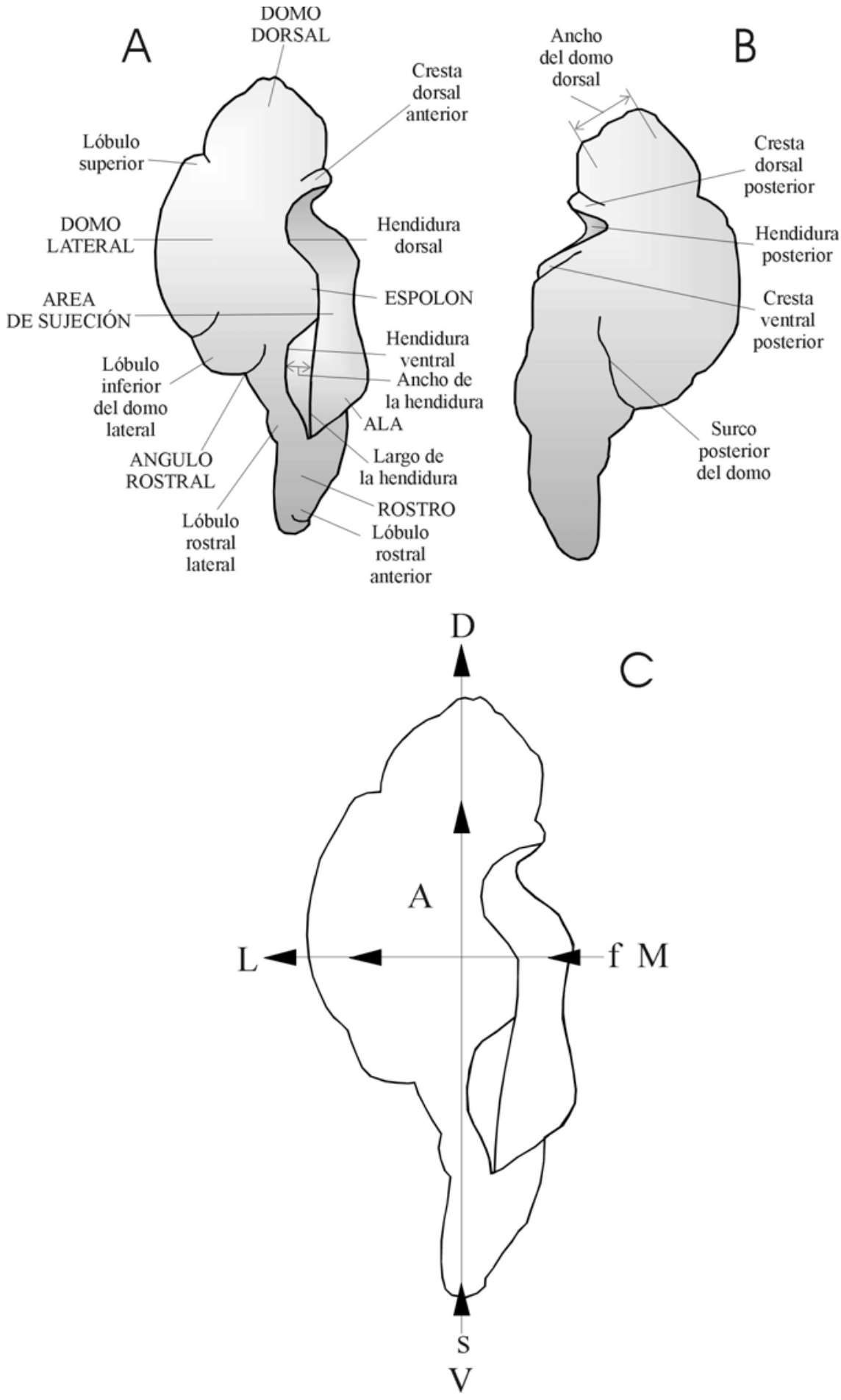

Figura 1

Esquemas del estatolito derecho mostrando las orientaciones y términos. (a) Vista anterior (Clarke 1978 y Leta 1982); (b) vista posterior (Clarke 1978 y Leta 1982); (c) planos de orientación (Lipinski et al. 1991). D:dorsal; V: ventral; L: lateral; A: anterior; M: medial; s: sagital; f: frontal.

Diagrams of the right statolith showing the positions and terms. (a) Anterior view (Clarke 1978 and Leta 1982); (b) posterior view (Clarke 1978 and Leta 1982); (c) planes of orientation (Lipinski et al. 1991). D:dorsal; V: ventral; L: lateral; A: anterior; M: medial; s: sagital; f: frontal. 
pequeña depresión visto por el lado anterior (figs. 2a y 2b). El domo lateral es de mayor tamaño, es elongado dorso-ventralmente en vista lateral y extendido con su superficie granulosa por el lado anterior (figs. 2a, 2b y 2c). El rostro es pequeño, algo alargado dorsoventralmente, terminado en punta en su lado ventral y aplanado latero-medialmente en vista anterior. La punta del rostro está levemente curvada hacia su lado anterior, y el ángulo rostral es obtuso $\left(130^{\circ}\right.$ - $\left.140^{\circ}\right)$ (figs. 2a, 2c y 2d). En el ala se observan: la hendidura dorsal, la hendidura ventral y el espolón por su lado anterior. La hendidura dorsal presenta una cresta anterior en su zona más dorsal. La hendidura ventral a diferencia de la hendidura dorsal no es tan profunda y no presenta cresta. El espolón es amplio y su parte ventral es redondeada. Por el lado posterior y en orientación dorsal es visible una hendidura que se llama hendidura posterior. En algunas muestras fue visible sobre esta hendidura, la cresta dorsal posterior (figs. 2a y 2b).
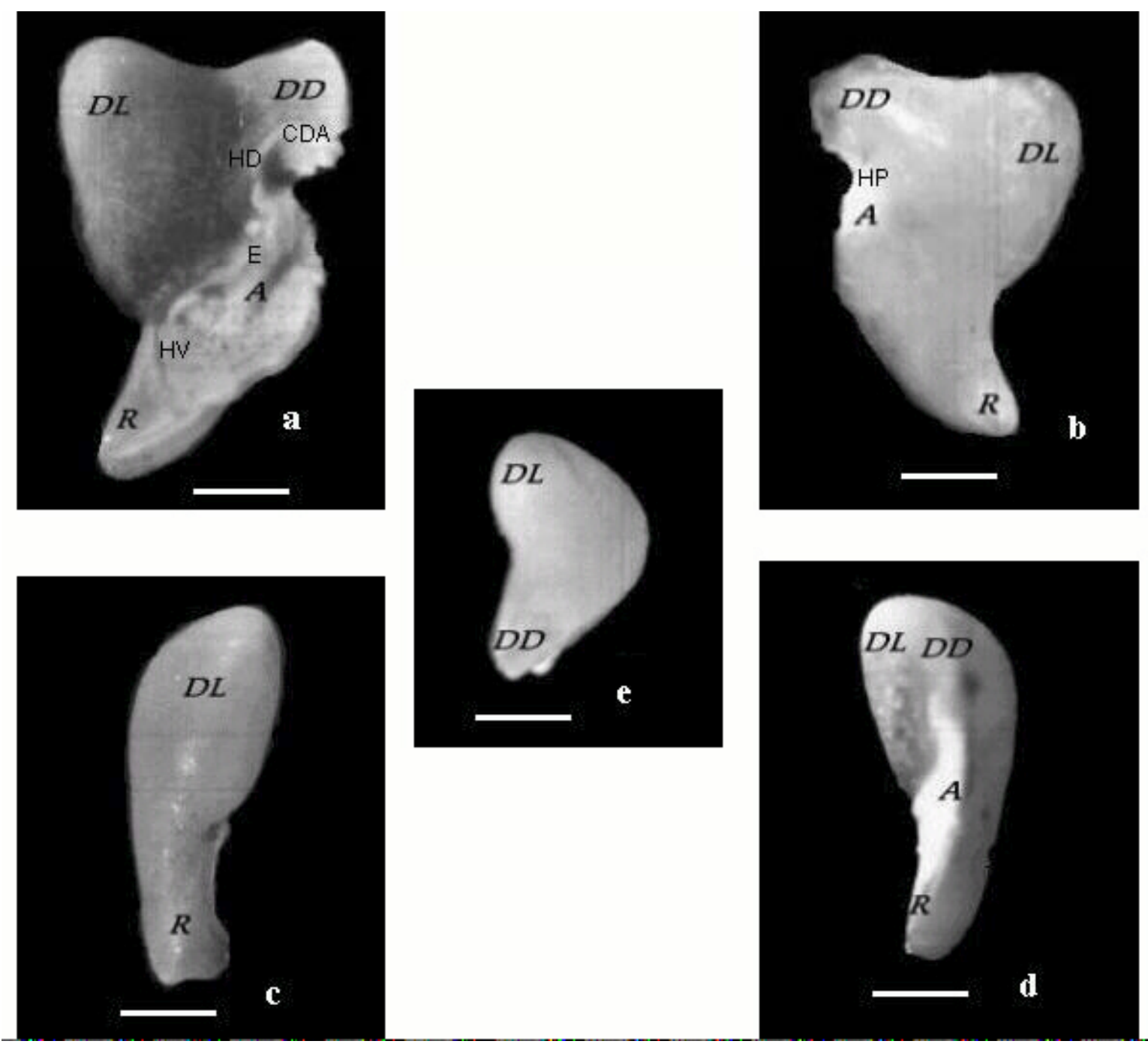

Figura 2

Estatolito derecho de Loligo gahi. (a) Vista anterior; (b) vista posterior; (c) vista lateral; (d) vista medial; (e) vista dorsal. DD: domo dorsal; DL: domo lateral; A: ala; R: rostro; HD: hendidura dorsal; E: espolón; HV: hendidura ventral; CDA: cresta dorsal anterior; HP: hendidura posterior, (Clarke 1978, Leta 1982 y Lipinski et al., 1991). Barra de escala: $350 \mu \mathrm{m}$.

Right statolith of Loligo gahi. (a) Anterior view; (b) posterior view; (c) lateral view; (d) medial view; (e) dorsal view. DD: dorsal dome; DL: lateral dome; A: wing; R: rostrum; HD: dorsal indentation; E: spur; HV: ventral indentation; CDA: anterior dorsal ridge; HP: posterior identation, (Clarke 1978, Leta 1982 and Lipinski et al., 1991). Scale bar: $350 \mu \mathrm{m}$. 


\section{Relación morfométrica entre la longitud del manto y el estatolito en Loligo gahi}

Una relación morfométrica importante a determinar en estudios de crecimiento en calamares es la proporcionalidad existente entre la longitud del manto y la estructura dura que se está usando para la determinación de la edad. Para esto se ajustaron distintos modelos a los datos de longitud del manto versus longitud del estatolito en el sentido domo dorsal - rostro (Clarke 1978, Hatfield 1991 y Arkhipkin \& Nekludova 1993), para el conjunto de individuos de la submuestra ( $\mathrm{n}=47)$ de Loligo gahi (fig. 3a). Los parámetros estimados por regresión para cada modelo se observan en la Tabla 1.
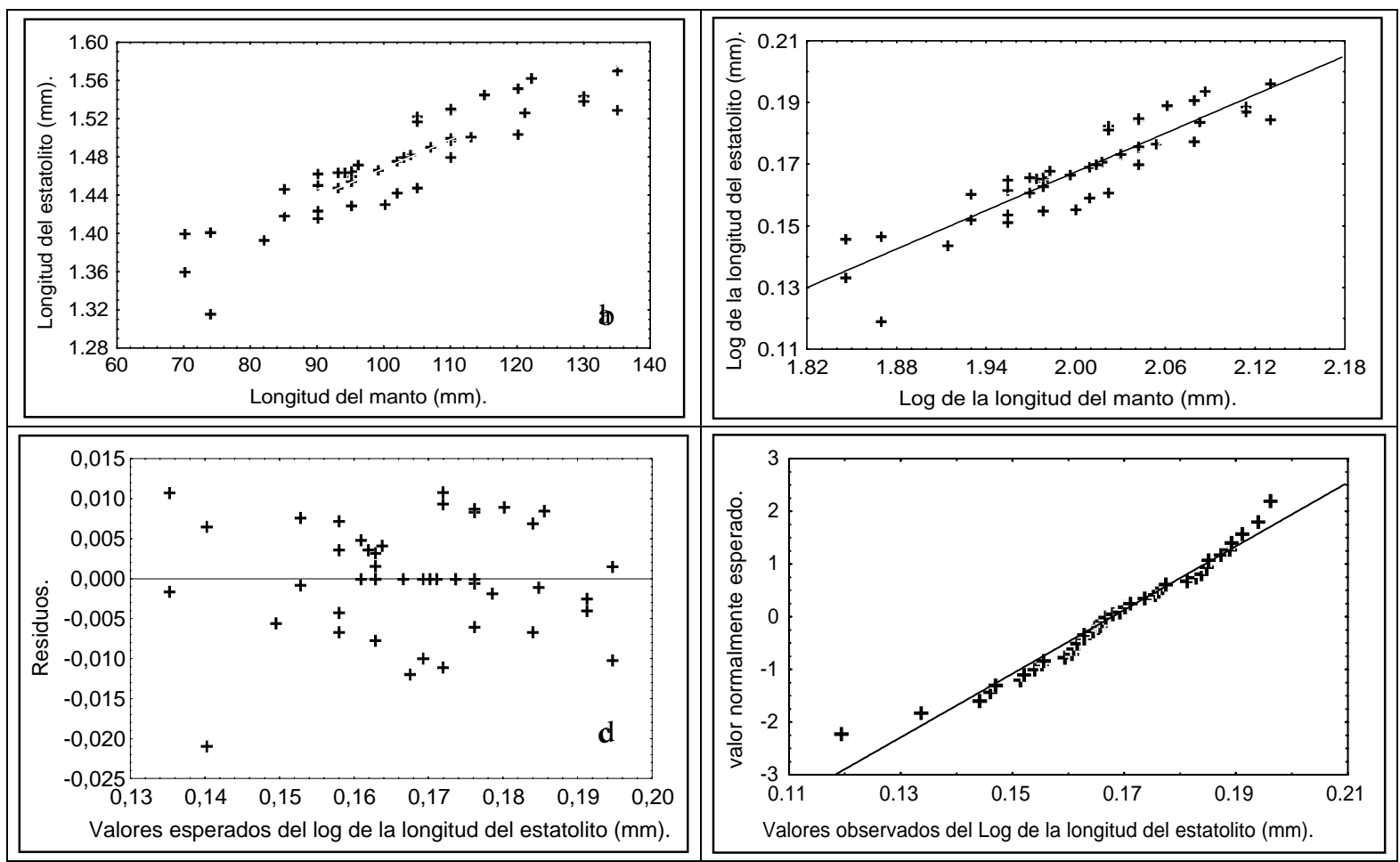

Figura 3

(a-d) Relación entre la longitud del manto y la longitud del estatolito.(a) gráfico de la longitud del manto v/s longitud del estatolito, (b) gráfico del log de la longitud del manto v/s log de la longitud del estatolito, (c) gráfico de los residuos, (d) gráfico de la probabilidad normal.

(a-d) Relationship between the mantle length and the statolith length.(a) graphic of the mantle length v/s the statolith length, (b) graphic of the $\log$ of the mantle length $\mathrm{v} / \mathrm{s}$ the log of the statolith length, (c) graphic of the residuals, (d) graphic of the normal probability.

Tabla 1

Parámetros estimados para la relación entre la longitud del estatolito (LE) y la longitud del manto (LM) para los distintos modelos en el calamar Loligo gahi. A: intercepto, B: pendiente, EE: error estandar, r: coeficiente de correlación, $\mathbf{r}^{2}$ : coeficiente de determinación.

Parameters estimated for the relationship between the statolith length (LE) and the mantle length (LM) for the differents models in the squid Loligo gahi. A: intercept, B: slope, EE: standard error, r: coefficient of correlation, $\mathrm{r}^{2}$ : coefficient of determination.

\begin{tabular}{|c|c|c|c|c|c|c|c|}
\hline MODELO & $\bar{A}$ & B & EE (A) & EE (B) & $\mathrm{r}$ & $\mathrm{R}^{2}$ & EE modelo \\
\hline $\mathrm{LE}=\mathrm{A}+\mathrm{B} * \mathrm{LM}$ & 1,165 & $3,03 * 10^{-3}$ & 0,0223 & $2,2 * 10^{-4}$ & 0,9006 & 0,807 & 0,0234 \\
\hline $\mathrm{LE}=\mathrm{A} * \mathrm{~B}^{\mathrm{LM}}$ & 1,1928 & 1,002 & $6,77 * 10^{-3}$ & $6,59 * 10^{-5}$ & 0,891 & 0,800 & $7,09 * 10^{-3}$ \\
\hline $\mathrm{LE}=\mathrm{A} * \mathrm{LM}^{\mathrm{B}}$ & 0,563 & 0,208 & $2,9 * 10^{-2}$ & $1,44 * 10^{-2}$ & 0,906 & 0,817 & $6,79 * 10^{-3}$ \\
\hline
\end{tabular}


La elección del modelo se basó en la comparación e interpretación de los estadísticos estimados, el análisis de los residuos, y el error estándar del modelo para la relación entre la longitud del manto y la longitud del estatolito. Así el modelo ajustado correspondió al geométrico cuya ecuación es (fig. 3b):

$$
\mathrm{LE}=0,5631^{*} \mathrm{LM}^{0,208}
$$

Donde : $\quad \mathrm{LE}=$ Longitud del estatolito.

$$
\text { LM = Longitud del manto }
$$

En el modelo ajustado se puede observar el buen grado de explicación de la variable longitud del estatolito por parte de la longitud del manto $\left(\mathrm{r}^{2}=0,81\right)$. Por otro lado los residuos demostraron homocedasticidad (fig.3c) y la interpretación del gráfico de probabilidad normal evidencia el cumplimiento del supuesto de normalidad (fig.3d). Es así entonces que el modelo ajustado cumple con los supuestos del análisis de regresión lineal.
Por último se realizó la prueba "t" de Student para determinar si el valor de la pendiente es distinto de 1 , lo cual indica que la relación de crecimiento entre la longitud del manto y la longitud del estatolito es alométrica (Ricker 1979), resultando en un rechazo de la hipótesis nula $(\mathrm{t}=-54,6<<\mathrm{t} \quad 0,05 ; 45=-2,02)$, o sea la pendiente es distinta de 1 .

\section{Relación de crecimiento entre la longitud del manto y el peso en Loligo gahi.}

Al igual que en el punto anterior en todo estudio de crecimiento es necesario determinar la relación de proporcionalidad entre la longitud del manto y el peso total del calamar. De esta forma se ajustaron distintos modelos a los datos de longitud del manto versus peso total para los individuos de la submuestra $(n=47)$ a través del análisis de regresión lineal (fig. 4a). En la Tabla 2 se observan los parámetros estimados para los diferentes modelos.
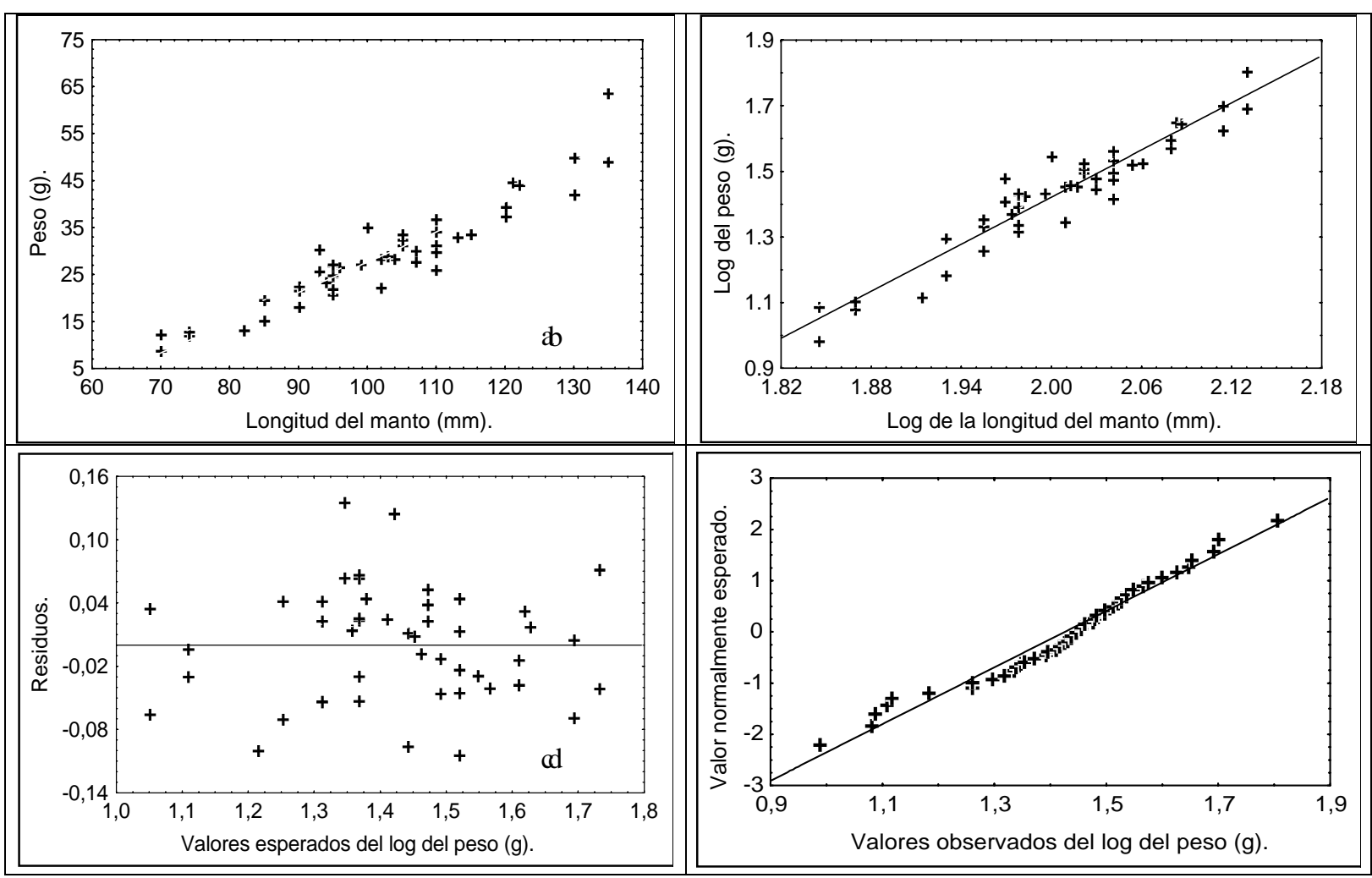

Figura 4

(a-d) Relación entre la longitud del manto y peso. (a) gráfico de la longitud del manto v/s el peso, (b) gráfico del log de la longitud del manto v/s log del peso, (c) gráfico de residuos (d) gráfico de probabilidad normal.

(a-d) Relationship between the mantle length and weight. (a) graphic of the mantle lenght v/s weight, (b) graphic of the log of the mantle length $\mathrm{v} / \mathrm{s}$ log of the weight, (c) graphic of the residuals, (d) graphic of the normal probability. 
Tabla 2

Parámetros estimados para la relación entre el peso y la longitud del manto para distintos modelos en el calamar Loligo gahi. A: intercepto, B: pendiente, EE: error estandar, $r$ : coeficiente de correlación, $\mathbf{r}^{2}$ : coeficiente de determinación.

Parameters estimated for the relationship between the weight and the mantle length for the differents models in the squid Loligo gahi. A: intercept, B: slope, EE: standard error, r: coefficient of correlation, $\mathrm{r}^{2}$ : coefficient of determination.

\begin{tabular}{|c|c|c|c|c|c|c|c|}
\hline MODELO & $\mathrm{A}$ & $\mathrm{B}$ & $\mathrm{EE}(\mathrm{A})$ & $\mathrm{EE}(\mathrm{B})$ & $\mathrm{r}$ & $\mathrm{R}^{2}$ & EE modelo \\
\hline $\mathrm{P}=\mathrm{A}+\mathrm{B} * \mathrm{LM}$ & $-36,7$ & $6,43 * 10^{-1}$ & 3,654 & $3,55^{*} 10^{-2}$ & 0,937 & 0,876 & 3,824 \\
\hline $\mathrm{P}=\mathrm{A} * \mathrm{~B}^{\mathrm{LM}}$ & 2,406 & 1,023 & $5,75 * 10^{-2}$ & $5,6 * 10^{-4}$ & 0,939 & 0,879 & $6,01 * 10^{-2}$ \\
\hline $\mathrm{P}=\mathrm{A} * \mathrm{LM}^{\mathrm{B}}$ & $4,41 * 10^{-4}$ & 2,388 & 0,233 & 0,116 & 0,950 & 0,900 & $5,46 * 10^{-2}$ \\
\hline
\end{tabular}

El criterio de selección del modelo se basó en la comparación e interpretación de los estadísticos estimados, en el análisis de los residuos, y en el error estándar del modelo. El modelo mejor ajustado y que representa la proporcionalidad existente entre el crecimiento en longitud y el crecimiento en peso de Loligo gahi es (fig 4b):

$$
\mathrm{P}=4,41 \times 10^{-4} * \mathrm{LM}^{2,388}
$$

Donde : $\quad \mathrm{P}=$ Peso

$$
\mathrm{LM}=\text { Longitud del manto }
$$

Luego de realizar el análisis del modelo se observó que existe un alto grado de explicación de la variable peso por parte de la longitud del manto, $\left(r^{2}=0,9\right)$. A partir del análisis de los residuos observamos que estos tienen un comportamiento homocedástico y el gráfico de probabilidad normal muestra que los datos se distribuyen normalmente. Es así entonces que se cumplen adecuadamente con los supuestos del análisis de regresión lineal (figs. 4c y 4d).

Por otro lado se realizó la prueba de “t” Student para determinar si el valor de la pendiente es distinta de 3 , lo cual indica que la relación de crecimiento entre la longitud del manto y el peso es del tipo alométrica (Ricker 1979, Forsythe \& van Heukelem 1987), lo que resultó en un rechazo de la hipótesis nula $(\mathrm{t}=-5,244<<\mathrm{t}$ 0,$05 ; 45=-2,02$ ), o sea la pendiente es distinta de 3 .

\section{Discusión}

\section{Morfología de los estatolitos de Loligo gahi}

Los estatolitos de Loligo gahi descritos son similares al de otros calamares de la familia Loliginidae (Natsukari et al. 1988, Arkhipkin \& Nekludova 1993). La identificación de la estructura morfológica que conforma el estatolito de Loligo gahi, en general no difiere del patrón básico descrito por Clarke (1978) y Lipinski et al. (1991) para un estatolito tipo en calamares. Sin embargo las diferencias más notables radican en la ausencia del lóbulo superior e inferior en el domo lateral, así como del lóbulo rostral en vista anterior y lateral, ni tampoco se observó por el lado posterior la cresta ventral.

Un aspecto importante a considerar en un próximo estudio son los cambios en la morfología de los estatolitos a través de todas las etapas ontogénicas, desde larvas a adultos de Loligo gahi, lo cual será util para establecer relaciones entre familias y géneros, así como para reconstruir la filogenia de diversos grupos. El contar con esta información permitirá aumentar la precisión de las determinaciones de especies de calamares en los estudios de ecología trófica y determinar el posible tamaño de la presa si se conoce la relación entre longitud del manto y longitud del estatolito.

\section{Relación entre la longitud del manto y el peso en Loligo gahi}

La relación de crecimiento observada entre la longitud del manto y el peso total en los ejemplares de Loligo gahi indica que es del tipo alométrico. Esto concuerda con lo observado por Arancibia \& Robotham (1984) quienes estimaron un valor de “b” para sexos combinados de 2,49, bastante cercano al valor estimado en el presente estudio ( $b=2,388)$.

En calamares neríticos de la familia Loliginidae a la que pertenece Loligo gahi, se observan valores de "b" menores de 2,7 (Holme 1974, Collins et al. 1995, Raya et al. 1999). En tanto que en calamares oceánicos de la familia Ommastrephidae se observan valores iguales o superiores a 2,7 (Garretón 1972, Mosalve 1985, Ehrhardt et al. 1983, Ragonese \& Jereb 1992, Arkhipkin \& Mikheev 1992, Paillamán et al. 1994, 
Belcari 1996, Hernández-García \& Castro 1998), con la excepción de Berriteuthis magister (Arkhipkin et al. 1996) y Onychoteuthis banki (Arkhipkin \& Nigmatullin, 1997) donde el valor de "b” es parecido al observado en calamares neríticos de la familia Loliginidae. Una explicación a las distintas relaciones longitud-peso para calamares neríticos (Loliginidae) y oceánicos (Ommastrephidae), es que serían indicativas de diferencias morfológicas adaptativas al comportamiento migratorio, ya que los calamares oceánicos son más grandes y robustos, con una gruesa musculatura de las paredes del manto; mientras que los calamares neríticos son más pequeños y ligeros, con delgadas paredes del manto. De esta manera no es de sorprender que especies de la familia Ommastrephidae aumenten en peso más rápidamente por unidad de longitud que las especies pertenecientes a la familia Loliginidae (Forsythe \& van Heukelem 1987, Belcari 1996). Este crecimiento alométrico observado en los individuos de Loligo gahi es, según Arkhipkin \& Mikheev (1992), HernándezGarcía \& Castro (1998), el resultado del crecimiento distinto de los brazos respecto al manto. Otro componente adicional de alometría es el desarrollo de las aletas, que se originan como pequeñas aletillas que crecen y se transforman en una estructura importante en adultos. La observación de crecimiento alométrico en este tipo de relaciones es usual ya que la mayoría de las especies exhiben un crecimiento alométrico del cuerpo a través del ciclo de vida, lo que significa que la forma y la densidad del cuerpo no permanecen constantes. En la mayoría de los animales la pendiente o exponente en la ecuación longitud-peso se encuentra entre 2,5 y 4 (Forsythe \& van Heukelem 1987).

Finalmente del análisis estadístico tenemos que nuestros datos muestran una alta significancia estadística en la explicación de la variabilidad del peso por parte de la longitud del manto $\left(r^{2}=0,9\right)$, lo que demuestra que el crecimiento en peso está altamente relacionado con el crecimiento en longitud del manto. A su vez del análisis de los residuos y del cuadrado medio del error se observa que se cumple con los supuestos de regresión y de esta forma el modelo matemático ajustado es el adecuado.

\section{Relación entre la longitud del manto y la longitud del estatolito en Loligo gahi.}

La relación de crecimiento entre la longitud del manto y la longitud del estatolito en Loligo gahi fue del tipo alométrico negativo, ya que el valor de "b" fue significativamente menor que $1(\alpha=0,05)$, reflejando el rápido crecimiento del manto. Este resultado coincide con lo observado por Arkhipkin \& Mikheev (1992) quienes señalan que la mayoría de los calamares presentan estatolitos caracterizados por una alometría negativa, es decir hay diferencias entre las tasas de crecimiento del calamar y las tasas de crecimiento del estatolito. Por otro lado nuestros resultados confirman lo observado en otras especies como Illex argentinus (Arkhipkin 1990, Brunetti \& Ivanovic 1990), Illex coindetti (Arkhipkin 1996), Onychoteuthis banski (Arkhipkin \& Nigmatullin 1997), Sthenoteuthis pteropus (Arkhipkin \& Mikhev 1992), Berryteuthis magister (Arkhipkin et al. 1996), Alloteuthis africana, A. subulata (Arkhipkin \& Nekludova 1993), Loligo vulgaris (Arkhipkin 1995, Rocha \& Guerra 1999) y Loligo forbesi (Collins et al. 1995, Rocha \& Guerra 1999), donde la relación entre las variables fue de una alometría negativa. La única excepción al ajuste geométrico observado en todos estos trabajos corresponde a Loligo forbesi (Collins et al. 1995), donde el ajuste fue exponencial. Por otro lado en Loligo gahi (Hatfield 1991) se establece este tipo de relación, pero se asigna como variable dependiente a la longitud del manto dando valores de "b" que no son comparables a los aquí obtenidos.

Con estos antecedentes el mejor modelo que se ajustó a los datos fue también el geométrico, donde gran parte de la variabilidad por parte de la longitud del estatolito es explicada por la longitud del manto $\left(r^{2}=0,817\right)$. El establecimiento de parámetros en este tipo de relaciones es importante ya que permiten inferir el crecimiento del estatolito a partir del crecimiento en longitud del manto y eventualmente estimar la longitud del calamar a diferentes edades como ha sido realizado en peces a través del proceso de retrocálculo (Francis 1990).

En un próximo trabajo sería de utilidad evaluar estadísticamente si existe un crecimiento diferencial por sexos a partir de las relaciones morfométricas y gravimetricas en el calamar patagónico (Loligo gahi).

\section{Agradecimientos}

Los autores agradecen al Sr Jorge Sierpe del Servicio Nacional de Pesca XII región por sus gestiones en la obtención de las muestras y a la Compañía Pesquera Pesca Chile por la donación de las mismas. Nuestros agradecimientos al Dr. Sergio Palma G., de la Universidad Católica de Valparaíso por su ayuda en la obtención de las microfotografías y a los evaluadores anónimos que contribuyeron a mejorar el manuscrito.

\section{Literatura Citada}

Arancibia H \& H Robotham.1984. Crecimiento y edad del calamar (Loligo gahi Orbigny) de la región austral de Chile (Teuthoidea-Loliginidae). Investigaciones Pesqueras 31: 71-79. 
Arkhikin A. 1990. Edad y crecimiento del calamar (IIlex argentinus). Frente Marítimo, 6(A): 25-35.

Arkhipkin A. 1991. Methods for cephalopods age and growth studies with emphasis on statolith ageing techniques. In: P.Jereb et al (eds), Squid age determination using statoliths p: 11-18. Italy Mazara del Vallo, NTR-ITPP.

Arkhipkin A. 1995. Age, growth and maduration of the european squid Loligo vulgaris (Myopsida,Loliginidae) on the west sahara shelf. Journal of the Marine Biological Association of the United Kingdom 75: 593-604.

Arkhipkin A. 1996. Geographical variation in growth and maduration of the squid Illex coindetii (Oegopsida, Ommastrephidae) off the north-west African coast. Journal of the Marine Biological Association of the United Kigdom 76: 1091-1106.

Arkhipkin A, V Bizikov, V Krylov \& K Nesis. 1996. Distribution, stock structure, and growth of the squid Berryteuthis magister (Berry, 1913) (Cephalopoda, Gonatidae) during summer and fall in the western Bering sea. Fishery Bulletin 94: 1-30.

Arkhipkin A \& A Mikheev. 1992. Age and growth of squid Stenoteuthis pteropus (Oegopsida: Ommastrephidae) from the central-east Atlantic. Journal of Experimental Marine Biology and Ecology 163: 261-276.

Arkhipkin A \& N Nekludova. 1993. Age, growth and maduration of the loliginid squids Alloteuthis africana and A. subulata on the west african shelf. Journal of the Marine Biological Association of the United Kingdom 73: 949-961.

Arkhipkin A \& C Nigmatullin. 1997. Ecology of the oceanic squid Onychoteuthis banski and the relationship between the genera Onychoteuthis and Chanoteuthis (Cephalopoda: Onychoteuthidae). Journal of the Marine Biological Association of the United Kigdom 77: 839-869.

Belcari P. 1996. Length-weight relationships in relation to sexual maduration of Illex coindetii (Cephalopoda: Ommastrephidae) in the northern Tyrrhenian sea (western Mediterranean). Scientia Marina 60: 379-384.

Brunetti N \& M Ivanovic. 1990. Morfología y morfometría de los estatolitos del calamar Illex argentinus. Frente marítimo 9: 53-61.

Caddy J. 1991. Daily rings on squid statoliths: an opportunity to test standard population models?. In: P.Jereb et al (eds), Squid age determination using statoliths p: 53-66. Italy Mazara del Vallo, NTR-ITPP.

Clarke M. 1975. First fossil records of cephalopod statoliths. Nature 257: 380-381.

Clarke M. 1978. The cephalopod statolith-an introduction to its form. Journal of the Marine Biological Association of the United Kingdom 58: 701-712.

Cochran W. 1971. Técnicas de Muestreo, 507p. Cecsa editores. México.

Collins M, G Burnell \& P Rodhouse. 1995. Age and growth of the squid Loligo forbesi (Cephalopoda: Loliginidae) in irish waters. Journal of the Marine Biological Association of the United Kigdom 75: 605-620.
Collins M \& G Pierce. 1996. Size selectivity in the diet of Loligo forbesi (Cephalopoda: Loliginidae). Journal of the Marine Biological Association of the United Kigdom 76: 1081-1090.

Csirke J. 1987. The patagonian fishery resources and the offshores fisheries in the southwest Atlantic. FAO Fisheries Tecnical Paper 286: 1-75.

Ehrhardt N, P Jacquemin, F García, G González, M López, C Ortiz. \& N Solis. 1983. On the fishery and biology of the giant squid Dosidicus gigas in the gulf of California, Mexico. In: Caddy J.F.(ed), Advances in assessment of world cephalopod resources. FAO Fisheries Technical Paper 231: 306-340.

FAO 1998. Fishery Statistics 82, 678 p.

Forsythe $\mathbf{J}$ \& $\mathbf{W}$ van Heukelem. 1987. Growth. In: P R Boyle (ed), Cephalopod Life Cycles, 2: 135-155. Academic Press, London.

Francis R. 1990. Back-calculation of fish lenght: a critical review. Journal of Fish Biology 36: 883-902.

Garretón M. 1972. Observaciones biológico-pesqueras en Dosidicus gigas D’Orbigny, 1835. Tesis departamento de Oceanología, 101p. U de Chile.

Hatfield E. 1991. Post-recluit growth of patagonian squid Loligo gahi (D’ Orbigny). Bulletin of Marine Science 49: 349-361.

Hernández-García V \& J Castro. 1998. Morphological variability in Illex coindetii (Cephalopoda: Ommastrephidae) along the north-west coast of Africa. Journal of the Marine Biological Association of the United Kigdom 78: 1259-1268.

Holme N. 1974. The biology of Loligo forbesi Steetrup (Mollusca : Cephalopoda) in the Plymounth area. Journal of the Marine Biological Association of the United Kingdom 54: 481-503.

Jackson G. 1994. Application and future potential of statolith increment analysis in squid and sepioids. Canadian Journal of Fisheries and Aquatic Science 51: 2612-2625.

Leta H. 1982. Descripción de la morfología de los estatolitos de tres especies de calamar (cephalopoda: Teuthoidea). Comunicaciones de la Sociedad Malacologica de Uruguay 43: 87-94.

Lipinski M, E Dawe \& Y Natsukari. 1991. Introduction to practical procedures of squid statoltihs. A laboratory manual. In: P.Jereb et al (eds), Squid age determination using statoliths, p: 77-82. Italy Mazara del Vallo, NTRITPP.

Monsalve R. 1985. Antecedentes biológico-pesqueros de la jibia (Dosidicus gigas). Estrategia para el desarrollo de pesquerías oceánicas en el área adyacente en la zona económica exclusiva. Apénidice 1. Documento técnico IFOP. AP85/38: 1-29.

Natsukari Y, T Nakanose \& K Oda. 1988. Age and growth of loliginid squid Photololigo edulis (Hoyle, 1885). Journal of Experimental Marine Biology and Ecology 116: 177-190. 
Paillaman A, M Rojas, R Bahamonde \& M Donoso. 1994. Pesca de investigación de jibia (Dosidicus gigas) en la zona económica exclusiva comprendida entre Arica y el paralelo $29^{\circ} 00^{\prime} \mathrm{S}$. Informe final. Instituto de Fomento Pesquero. 60p.

Pineda S, D Hernández \& N Brunetti. 1998. Statolith comparision of two south-west Atlantic loliginid squid: Loligo sanpaulensis and Loligo gahi. South African Journal of Marine Science. 20: 347-354.

Ragonese S \& P Jereb. 1992. Length-weight relationships of IIlex coindetti verany, 1839 (Mollusca: Cephalopoda) in the Sicilian channel. Oebalia 28: 17-24.

Raya C, M Balguerías, M Fernández - Núñez \& G Pierce.1999. On reproduction and age of the squid Loligo vulgaris from the Saharian bank (north-west African coast). Journal of the Marine Biological Association of the United Kigdom 79: 111-120.

Ricker W. 1979. Growth rates and models. In: W Hoa et al. (eds), Fish Physiology Vol III, p: 677-743. Academic Press, London.
Rocha F \& A Guerra 1999. Age and growth of two sympatric squid Loligo vulgaris and Loligo forbesi, Galician waters (north-west Spain). Journal of the Marine Biological Association of the United Kigdom 79: 697-707.

Rodhouse P \& E Hatfield. 1990. Age determination in squid using statolith growth increments. Fisheries Research 8: 323-334.

Roper C, M Sweeney \& C Nauen. 1984. FAO Species catalogue. Cephalopods of the world. Ann annotated and ilustrated catalogue of species of interest to Fisheries. FAO Fishery Synopsis 3 (125): 277p.

Vega M, F Rocha \& C Osorio. 2001. Morfometría comparada de los estatolitos del calamar Loligo gahi d' Orbigny, 1835 (Cephalopoda: Loliginidae) del norte de Perú e islas Falkland. Investigaciones Marinas 29: 3-9. 